\title{
Fabrication of nanoscale antidot arrays and magnetic observations using $x$-ray photoemission electron microscopy
}

\author{
L. J. Heyderman ${ }^{\text {a) }}$ and H. H. Solak \\ Laboratory for Micro- and Nanotechnology, Paul Scherrer Institut, CH-5232 Villigen PSI, Switzerland
}

F. Nolting and C. Quitmann

Swiss Light Source, Paul Scherrer Institut, CH-5232 Villigen PSI, Switzerland

(Presented on 6 January 2004)

\begin{abstract}
Antidot arrays in thin cobalt films have been fabricated with periods ranging from $2 \mu \mathrm{m}$ down to $100 \mathrm{~nm}$ and with different size to separation ratios. The resulting magnetic domains were observed with a photoemission electron microscope using x-rays. For periods larger than $1 \mu \mathrm{m}$, we observe magnetic domain configurations which substantiate micromagnetic calculations found in the literature. At smaller periods the domain configuration changes dramatically resulting in chains of magnetic domains running parallel to the intrinsic hard axis. (c) 2004 American Institute of Physics.
\end{abstract}

[DOI: $10.1063 / 1.1669117]$

\section{INTRODUCTION}

Magnetic thin films with periodic arrays of nonmagnetic inclusions, referred to as antidots, have been suggested as a possible candidate for ultrahigh density storage. ${ }^{1}$ The demagnetizing fields associated with the antidots tend to align the magnetization parallel to the edge of the antidot and for a magnetic thin film with uniaxial anisotropy, the resulting domain configuration contains regions with remanent magnetization parallel to the intrinsic hard axis. These regions can be used to store bits of information. ${ }^{1,2}$ Antidot arrays are also of interest because they significantly modify the switching characteristics in magnetic thin films and it is possible to tailor both the switching fields and magnetoresistance behavior by changing the size, separation, and the lattice symmetry and orientation of the antidots. ${ }^{3-6}$

In the present study, we have fabricated square lattice antidot arrays with periods ranging from $2 \mu \mathrm{m}$ down to $100 \mathrm{~nm}$ and observed the magnetic domain configurations using x-ray photoemission electron microscopy (PEEM). ${ }^{7}$ Being nonintrusive and providing direct imaging of the magnetization orientations, this observation technique has a significant advantage over magnetic force microscopy studies ${ }^{8,9}$ and in contrast to Lorentz microscopy, ${ }^{10}$ no special preparation of the sample substrate is required.

\section{EXPERIMENT}

The antidot arrays were fabricated with electron beam lithography using a lift-off process to transfer the pattern written in a poly(methylmethacrylate) resist on a $\mathrm{Si}(001)$ substrate into $10-\mathrm{nm}$-thick polycrystalline cobalt films. The films were deposited by dc-magnetron sputtering and have a 1-nm-thick aluminum capping layer to prevent oxidation of the magnetic film. We produced various antidot separations, $d$, with values ranging from just below the antidot period, $p$,

a) Author to whom correspondence should be addressed: electronic mail: laura.heyderman@psi.ch down to $50 \mathrm{~nm}$. This provides a range of $w / d$, where $w$ is the antidot size. A scanning electron microscope (SEM) image of the smallest period antidot array, $p=100 \mathrm{~nm}$, is shown Fig. 1. In the image there are three positions where the antidot has not been removed during the lift-off process indicating that we are close to the minimum size that can be produced using this lithography process. Due to the limited resolution of fabrication process, the antidots change in geometry from square to round holes when $w \leqslant 150 \mathrm{~nm}$. Hysteresis loop measurements were carried out on a reference sample with a continuous cobalt film of thickness, $10 \mathrm{~nm}$, grown using the same deposition system. The continuous film displayed a uniaxial anisotropy, which is likely to be due to a small field (20-30 Oe) present during sputter deposition. The anisotropy field has a value of about 50 Oe and an easy axis coercivity of about 25 Oe.

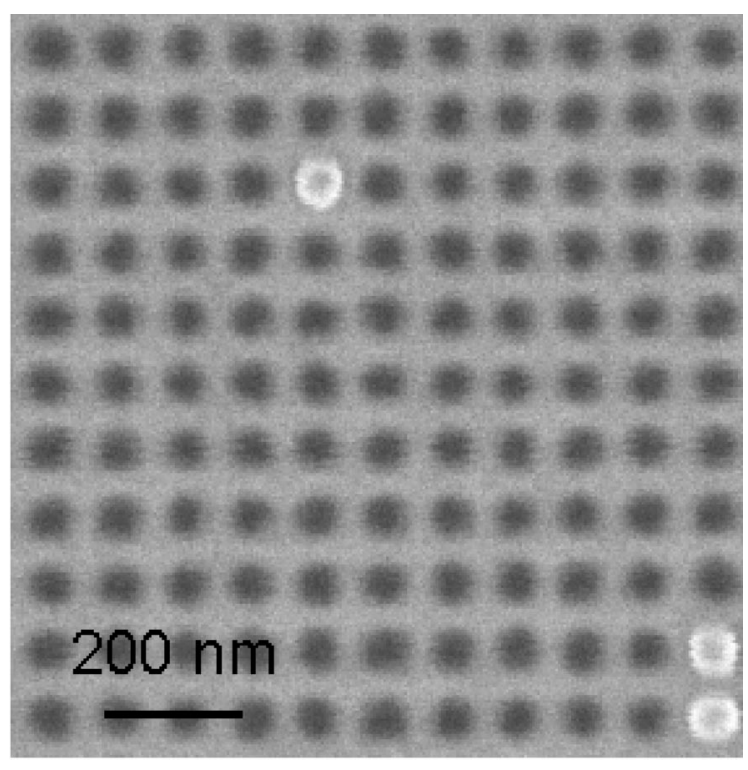

FIG. 1. SEM image of smallest antidot array with $100 \mathrm{~nm}$ period $(d=w=50 \mathrm{~nm})$. 
The imaging was carried out at the SIM beamline ${ }^{11}$ at the Swiss Light Source, Paul Scherrer Institut, with an Elmitec PEEM. ${ }^{12}$ Employing x-ray magnetic circular dichroism (XMCD), the magnetic domains are imaged by tuning the x-ray energy to the Co L edge. Dividing two images taken with left and right circular polarized light leads to an XMCD image where the intensity is a measure of the angle between the circular x-ray polarization vector, labeled as magnetization sensitivity direction in the figures, and the magnetization in the domains. ${ }^{13}$ Ferromagnetic domains with magnetization parallel or antiparallel to the polarization vector appear dark (black) or light (white) in the XMCD image and oppositely magnetized domains with magnetization perpendicular to the polarization vector will both have the same intermediate (gray) contrast.

\section{DOMAIN OBSERVATIONS}

We first investigate the magnetic domain configurations in the as-grown antidot arrays with $p=2 \mu \mathrm{m}$, and $w / d$ ranging from 0.08 to 3.65 . The extent of modification of the thin film magnetic domain structure increases with increasing $w / d$. For $w / d<0.9$, we observe "whisker domains" which form at the edges of the antidots to reduce the demagnetization energy. For $w / d>0.4$, the demagnetizing field associated with the antidots is large enough to induce the formation of additional domains which run diagonally between antidots. Both whisker and diagonal domains can be seen in Fig. 2(a). When $w$ approaches $d$, we observe a change in the magnetic domain configuration to a checked domain pattern with three domain contrasts, which is periodic in two dimensions and commensurate with the antidot array [Fig. 2(b)]. The whisker, diagonal and checked domain configurations shown in Figs. 2(a) and 2(b) are comparable to the domain configurations predicted by micromagnetic theory. ${ }^{4-16}$

We now concentrate on the antidot arrays with $d \approx w$ and observe what happens in the as-grown magnetic state as the period decreases. Checked domains with two-dimensional periodicity are also seen for a period of $1 \mu \mathrm{m}$. However, at a period of $400 \mathrm{~nm}$ [Fig. 2(c)], we observe a completely different magnetic configuration: chains of domains running parallel to the intrinsic hard axis [Fig. 3(b)]. These have different lengths corresponding to multiples of the antidot period and, unexpectedly, the magnetization in the dark and light areas is approximately parallel to the intrinsic hard axis. Such magnetic domain chains are observed down to an antidot period of $200 \mathrm{~nm}$, which is close to the current resolution limit of $150 \mathrm{~nm}$ of the PEEM microscope. The dark contrast appears to be a result of chains of domains which diagonally join antidots. This is reminiscent of the diagonal domains in Fig. 2(a), and we believe that similar magnetic energy principles govern their formation. Future micromagnetic simulations should clarify the mechanisms behind the formation of these domains. If the final length of the chains is not defined by the presence of defects, it may be possible to switch individual adjacent regions to change the chain length at will, so providing a method of information storage with the recording track parallel to the chain.

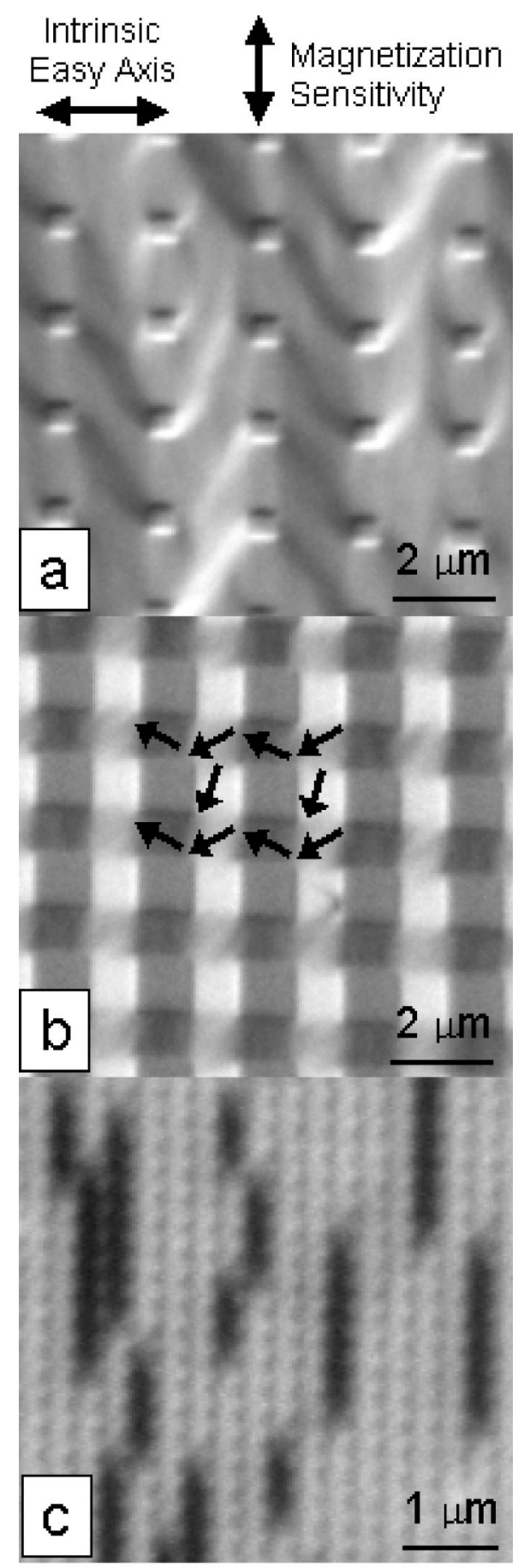

FIG. 2. Magnetic domain configurations in antidot arrays with period and ratio of antidot size to separation (a) $p=2 \mu \mathrm{m}$ and $w / d=0.38$, (b) $p=2 \mu \mathrm{m}$ and $w / d=1.41$ and (c) $p=300 \mathrm{~nm}$ and $w / d=1$. The black arrows in (b) indicate the orientation of the magnetization.

We then observe what happens following demagnetization which is carried out by application of a $50 \mathrm{~Hz}$ acmagnetic field with an amplitude slowly reduced from 150 Oe to zero. For an antidot array with $p=2 \mu \mathrm{m}$ and $w / d=0.90$, we observe a change in the magnetic configuration. Two domains with opposite magnetizations ( $D$ and $E$ ) are now present and separated by a domain boundary pinned by the antidots. This results in small sections of the wall pinned diagonally between two antidots, for example, in the 


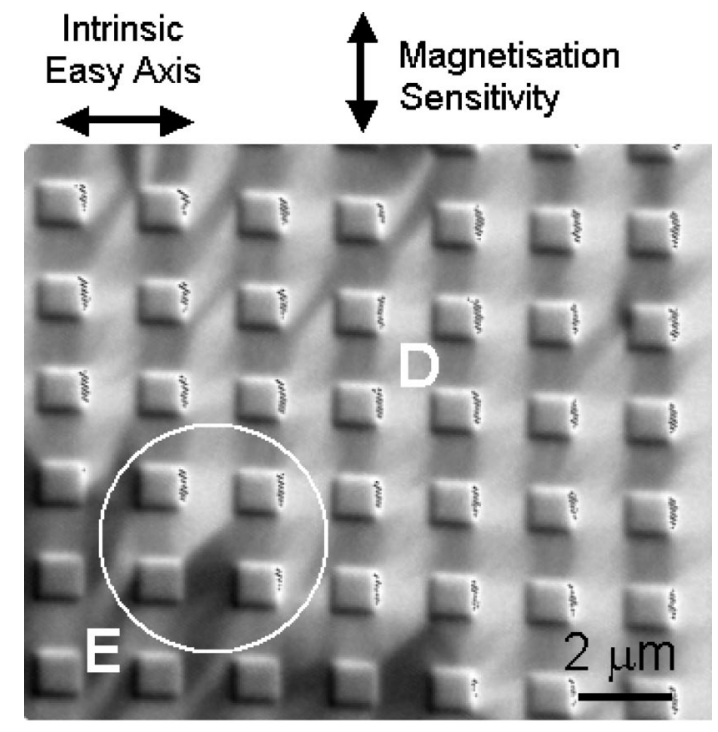

FIG. 3. Magnetic domain configuration in antidot array with period, $p=2 \mu \mathrm{m}$ and ratio of antidot size to separation, $w / d=0.90$, following application of a $50 \mathrm{~Hz}$ ac-demagnetizing field with amplitude of $150 \mathrm{Oe}$.

region delimited by the circle. On application of a $150 \mathrm{Oe}$ demagnetizing field to antidot arrays with $p \leqslant 400 \mathrm{~nm}$, the chain domains appear to be stable.

\section{CONCLUSION}

We have investigated the modification of the magnetization distribution in thin magnetic films containing antidot arrays with a wide range of $p$ and $d / w$ using X-ray PEEM. The observed magnetic domain configurations for $p \geqslant 1 \mu \mathrm{m}$ agree with the predictions of micromagnetic calculations. ${ }^{14-16}$ A dramatic change in the magnetic domain configuration at $p=400 \mathrm{~nm}$ occurs resulting in chains of coupled magnetic domains running parallel to the intrinsic hard axis. These results could prove to be important for tailoring of magnetic thin film properties and storage of magnetic information.

\section{ACKNOWLEDGMENTS}

The authors would like to thank Luis López-Díaz, Salamanca University, for valuable advice and discussions, and at the Paul Scherrer Institut: Michael Horisberger for the sputter deposition, and Christian David for his support with electron beam lithography. Part of this work was performed at the SLS, Paul Scherrer Institut, Villigen, Switzerland.

${ }^{1}$ R. P. Cowburn, A. O. Adeyeye, and J. A. C. Bland, J. Magn. Magn. Mater. 173, 193 (1997).

${ }^{2}$ R. P. Cowburn, A. O. Adeyeye, and J. A. C. Bland, Appl. Phys. Lett. 70, 2309 (1997).

${ }^{3}$ A. O. Adeyeye, J. A. C. Bland, and C. Daboo, Appl. Phys. Lett. 70, 3164 (1997).

${ }^{4}$ Z. L. Xiao, Y. C. Han, U. Welp, H. H. Wang, V. K. Vlasko-Vlasov, W. K. Kwok, D. J. Miller, J. M. Hiller, R. E. Cook, G. A. Willing, and G. W. Crabtree, Appl. Phys. Lett. 81, 2869 (2002).

${ }^{5}$ M. B. A. Jalil, S. L. A. Phoa, S. L. Tan, and A. O. Adeyeye, IEEE Trans. Magn. 38, 2556 (2002).

${ }^{6}$ I. Ruiz-Feal, L. Lopez-Diaz, A. Hirohata, J. Rothman, C. M. Guertler, J. A. C. Bland, L. M. Garcia, J. M. Torres, J. Bartolome, F. Bartolome, M. Natali, D. Decanini, and Y. Chen, J. Magn. Magn. Mater. 242-245, 597 (2002).

${ }^{7}$ J. Stöhr, H. A. Padmore, S. Anders, T. Stammler, and M. R. Scheinfein, Surf. Rev. Lett. 5, 1297 (1998).

${ }^{8}$ C. T. Yu, H. Jiang, L. Shen, P. J. Flanders, and G. J. Mankey, J. Appl. Phys. 87, 6322 (2000).

${ }^{9}$ P. Vavassori, G. Gubbiotti, G. Zangari, C. T. Yu, H. Yin, H. Jiang, and G. J. Mankey, J. Appl. Phys. 91, 7992 (2002).

${ }^{10}$ A. Y. Toporov, R. M. Langford, and A. K. Petford-Long, Appl. Phys. Lett. 77, 3063 (2000).

${ }^{11}$ C. Quitmann, U. Flechsig, L. Patthey, T. Schmidt, G. Ingold, M. Howells, M. Janousch, and R. Abela, Surf. Sci. 480, 173 (2001).

${ }^{12}$ T. Schmidt, S. Heun, J. Slezak, J. Diaz, K. C. Prince, G. Lilienkamp, and E. Bauer, Surf. Rev. Lett. 5, 1287 (1998).

${ }^{13}$ A. Scholl, H. Ohldag, F. Nolting, J. Stöhr, and H. A. Padmore, Rev. Sci. Instrum. 73, 1362 (2002).

${ }^{14}$ L. Lopez-Diaz, L. Torres, and J. I. Iniguez, J. Magn. Magn. Mater. 196197, 805 (1999).

${ }^{15}$ L. Torres, L. Lopez-Diaz, and J. Iniguez, Appl. Phys. Lett. 73, 3766 (1998).

${ }^{16}$ L. Torres, L. Lopez-Diaz, O. Alejos, and J. Iniguez, J. Appl. Phys. 85, 6208 (1999). 